\title{
VEGF $_{164}$-mediated Inflammation Is Required for Pathological, but Not Physiological, Ischemia-induced Retinal Neovascularization
}

\author{
Susumu Ishida, ${ }^{1,3}$ Tomohiko Usui, ${ }^{1,4}$ Kenji Yamashiro, ${ }^{1,5}$ Yuichi Kaji, ${ }^{1,4}$ \\ Shiro Amano, ${ }^{4}$ Yuichiro Ogura, ${ }^{6}$ Tetsuo Hida,${ }^{7}$ Yoshihisa Oguchi, ${ }^{3}$

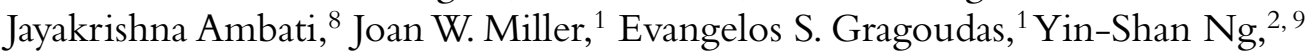 \\ Patricia A. D'Amore, ${ }^{2}$ David T. Shima, ${ }^{9}$ and Anthony P. Adamis ${ }^{1,9}$ \\ ${ }^{1}$ Angiogenesis Laboratory, Department of Ophthalmology, Massachusetts Eye and Ear Infirmary and \\ ${ }^{2}$ Department of Pathology and Ophthalmology, Schepens Eye Research Institute,Harvard Medical School, Boston, \\ $M A 02114$ \\ ${ }^{3}$ Department of Ophthalmology, Keio University School of Medicine, Tokyo 160-8582, Japan \\ ${ }^{4}$ Department of Ophthalmology, Faculty of Medicine, University of Tokyo, Tokyo 113-8655, Japan \\ ${ }^{5}$ Department of Ophthalmology and Visual Sciences, Kyoto University Graduate School of Medicine, Kyoto \\ 606-8507, Japan \\ ${ }^{6}$ Department of Ophthalmology, Nagoya City University Medical School, Nagoya 467-8601, Japan \\ ${ }^{7}$ Kyorin Eye Center, Mitaka 181-8611, Japan \\ ${ }^{8}$ Department of Ophthalmology, University of Kentucky, Lexington, KY 40517 \\ ${ }^{9}$ Eyetech Research Center, Woburn, MA 01801
}

\begin{abstract}
Hypoxia-induced VEGF governs both physiological retinal vascular development and pathological retinal neovascularization. In the current paper, the mechanisms of physiological and pathological neovascularization are compared and contrasted. During pathological neovascularization, both the absolute and relative expression levels for $\mathrm{VEGF}_{164}$ increased to a greater degree than during physiological neovascularization. Furthermore, extensive leukocyte adhesion was observed at the leading edge of pathological, but not physiological, neovascularization. When a VEGF $_{164}$-specific neutralizing aptamer was administered, it potently suppressed the leukocyte adhesion and pathological neovascularization, whereas it had little or no effect on physiological neovascularization. In parallel experiments, genetically altered $\mathrm{VEGF}_{164}$-deficient $\left(\mathrm{VEGF}^{120 / 188}\right.$ ) mice exhibited no difference in physiological neovascularization when compared with wild-type $\left(\mathrm{VEGF}^{+/+}\right)$controls. In contrast, administration of a VEGFR-1/Fc fusion protein, which blocks all VEGF isoforms, led to significant suppression of both pathological and physiological neovascularization. In addition, the targeted inactivation of monocyte lineage cells with clodronateliposomes led to the suppression of pathological neovascularization. Conversely, the blockade of $\mathrm{T}$ lymphocyte-mediated immune responses with an anti-CD2 antibody exacerbated pathological neovascularization. These data highlight important molecular and cellular differences between physiological and pathological retinal neovascularization. During pathological neovascularization, VEGF $_{164}$ selectively induces inflammation and cellular immunity. These processes provide positive and negative angiogenic regulation, respectively. Together, new therapeutic approaches for selectively targeting pathological, but not physiological, retinal neovascularization are outlined.
\end{abstract}

\section{Introduction}

Key words: retina $\bullet$ angiogenesis $\bullet \mathrm{VEGF} \bullet$ leukocyte $\bullet$ immunity
Retinal neovascular diseases such as retinopathy of prematurity and proliferative diabetic retinopathy are the major

\footnotetext{
S. Ishida, T. Usui, and K. Yamashiro contributed equally to this work. Address correspondence to Anthony P. Adamis, Eyetech Research Center, 42 Cummings Park, Woburn, MA 01801. Phone: 781-9353937; Fax: 781-935-9083; email: tony.adamis@eyetk.com
}

causes of neonatal and adult blindness. During both physiological retinal vascular development (1) and pathological proliferative retinopathy (2), new blood vessel growth is regulated by vascular endothelial growth factor (VEGF), a hypoxia-induced endothelial cell-specific mitogen produced in ischemic retinal cells. Although both types of 
neovascularization stem from retinal ischemia, there is an essential difference in the direction of vessel growth during physiological and pathological neovascularization. In the former, new vessels extend from the optic disc toward the peripheral avascular retina, and follow the guidance of VEGF-expressing retinal astrocytes (2). During pathological neovascularization, although ischemic retinal astrocytes and neurons express VEGF (1), the new vessels invade the vitreous cavity. This ectopic neovascularization leads to fibrovascular proliferation, resulting in visionthreatening complications, such as vitreous hemorrhage and traction retinal detachment. Coincident with the pathological neovascularization, the once-vascularized and newly ischemic central areas of the retina are reinvested with normal-appearing blood vessels, a process termed "revascularization." When reagents that block all VEGF isoforms are used, this compensatory intraretinal revascularization is suppressed together with the pathological neovascularization (3). Thus, ophthalmologists await the establishment of a new therapy that selectively targets pathological neovascularization, while sparing compensatory revascularization.

The current paper demonstrates that the differential expression of $\mathrm{VEGF}_{164}$ results in leukocyte adhesion at the leading edge of pathological, but not physiological, neovascularization. Furthermore, differential functions for the various leukocyte subpopulations recruited to the sites of neovascularization are demonstrated here. As a result, new mechanistic differences are uncovered at the molecular and cellular levels between the physiological and pathological neovascularization.

\section{Materials and Methods}

Rat Model of Proliferative Retinopathy. All animal experiments followed the Association for Research in Vision and Ophthalmology guidelines and were approved by the Animal Care Committee of Massachusetts Eye and Ear Infirmary. Long-Evans rats (Charles River Laboratories) were used. Postnatal day zero (P0) rats with their nursing mothers were maintained for 10 full days in $80 \%$ oxygen, interrupted daily by $30 \mathrm{~min}$ in room air followed by a progressive return to $80 \%$ oxygen, to induce an avascular retina. On P10 (D0), they were placed in room air for an additional $7 \mathrm{~d}$ (D7) to induce retinal neovascularization.

ELISA for VEGF. After sacrifice with an overdose of anesthesia, the eyes were immediately enucleated. The retina was carefully isolated, placed into $150 \mu \mathrm{l}$ of lysis buffer, and sonicated. The lysate was centrifuged at $14,000 \mathrm{rpm}$ for $15 \mathrm{~min}$ at $4^{\circ} \mathrm{C}$, and the VEGF levels in the supernatant were determined with the Quantikine mouse VEGF ELISA kit (R\&D Systems) according to manufacturer's protocol. The assay recognizes all VEGF isoforms.

Reverse Transcription (RT)-PCR for VEGF. Total RNA was isolated from the retina and peripheral monocytes with TRIzol reagent (Life Technologies), and cDNA was produced using reverse transcriptase (SuperScript II; Invitrogen). The primer sequences for GAPDH and rat VEGF were 5'-CCATGGAGAAGGCTGGGG-3' (sense) and 5'-CAAAGTTGTCATGGATGACC-3' (antisense) for GAPDH and 5'-ACCTCCACCATGCCAAGT-3' (sense) and 5'-TAGTTCCCGAAACCCTGA-3' (antisense) for VEGF. Analysis of RT-PCR data was performed using the intensity ratios of $\mathrm{VEGF}_{164} / \mathrm{VEGF}_{120}$ in each lane.

Lectin Labeling of Retinal Vasculature and Adherent Leukocytes. The retinal vasculature and adherent leukocytes were imaged by perfusion-labeling with FITC-coupled Concanavalin A lectin (Con A; Vector Laboratories) as described previously (4). The flat mounts were imaged using two epifluorescence microscopes (models DM RXA and MZ FLIII; Leica), each possessing a different range of magnification.

Intravitreous Injection of Anti-VEGF ${ }_{165}$ Aptamer (EYE001). After deep anesthesia, an eyelid fissure was created with a fine blade to expose the eyeball. Intravitreous injections were performed by inserting a 33-gauge double caliber needle (Ito Corporation) under an operating microscope. Animals received intravitreous injections of $1 \mu \mathrm{l}$ of sterile PBS containing $0.5 \mathrm{nmol}$ of a pegylated anti-VEGF 165 aptamer (EYE001; Eyetech Pharmaceuticals) or polyethylene glycol (PEG) control. Retinal neovascularization and leukocyte adhesion were evaluated $7 \mathrm{~d}$ after administration in D0 (P10) retinopathy rats and P3 normal neonates.

The anti-VEGF 165 aptamer is an oligonucleotide that binds to the exon 7-encoded domain of human $\mathrm{VEGF}_{165}$ protein with high specificity and affinity. The oligonucleotide is conjugated to a 40-kD PEG moiety to increase its half-life. The aptamer does not bind to $\mathrm{VEGF}_{120(121)}$ and efficiently neutralizes $\mathrm{VEGF}_{164}$ in rats.

Intravitreous Injection of a VEGFR-1/Fc Fusion Protein. Animals received $1-\mu 1$ intravitreous injections of sterile PBS containing $1 \mu \mathrm{g}$ of a mouse VEGFR-1/Fc chimera (R\&D Systems) or an isotype $F_{c}$ control (R\&D Systems). The fusion protein blocks all VEGF isoforms. A maximal effect dosage (1 $\mu \mathrm{g}$ per eye) was determined and used for comparison with the maximal effect dosage of the anti-VEGF 165 aptamer.

Generation of VEGF $164^{- \text {deficient }}\left(V_{\left.E G F^{120 / 188}\right)}\right.$ Mice. VEGF $^{+/ 120}$ male and $\mathrm{VEGF}^{+/ 188}$ female mice, generated previously (5) via targeted mutagenesis with Cre/loxP-mediated site-specific recombination in embryonic stem cells, were mated to each other. Sired neonates were genotyped as described previously (5) to select VEGF $_{164}$-deficient $\left(V_{E G F}{ }^{120 / 188}\right.$ ) mice and age-matched wild-type control $\left(\mathrm{VEGF}^{+/+}\right)$mice for analysis at $\mathrm{P} 10$.

CD13, CD8, and CD25 Immunofluorescence. Adherent leukocytes were labeled with rhodamine-coupled Con A as described above in the fourth paragraph of Materials and Methods. Retinal flat mounts were permeabilized with $0.5 \%$ Triton X (Sigma-Aldrich) in PBS for $24 \mathrm{~h}$ and nonspecific binding was blocked with $1 \%$ BSA. The retinas were incubated with a FITCconjugated mouse monoclonal antibody against CD13 (clone WM15, 1:100; Research Diagnostics), CD8 (clone OX-8, 1:100; Research Diagnostics), or CD25 (clone OX-39, 1:100; Research Diagnostics) overnight at $4^{\circ} \mathrm{C}$.

Isolation and Hypoxic Stimulation of Monocytes. Blood was drawn from D7 rats with retinopathy before sacrifice. The monocytes were purified by positive selection via magnetic cell sorting (MACS) using MicroBeads conjugated with a monoclonal antirat MHC-II antibody (clone OX-6; Miltenyi Biotec) according to the manufacturer's instructions. The isolated monocytes were seeded at a concentration of $2.5 \times 10^{6}$ cells per dish and stimulated for $8 \mathrm{~h}$ at $37^{\circ} \mathrm{C}$ with 1 or $21 \%$ oxygen in a humidified airtight chamber (Modular Incubator Chamber; Billups-Rothenberg). The incubated cells were collected and processed for RT-PCR for VEGF as described above in the third paragraph of Materials and Methods.

Intravitreous Administration of Clodronate-liposomes. Clodronate (dichloromethylene diphosphonate) was a gift from Roche 
Diagnostics. Animals with retinopathy received $1-\mu$ l intravitreous injections of clodronate- or PBS-liposomes on D0 (P10). The degree of retinal neovascularization was evaluated $7 \mathrm{~d}$ later.

Systemic CD2 Blockade. Rats with retinopathy received intraperitoneal injections of $5 \mathrm{mg} / \mathrm{kg} /$ day of a mouse anti-rat CD2 neutralizing antibody (clone OX-34; Research Diagnostics) or a mouse isotype nonimmune antibody (R\&D Systems). The reagents were injected for seven consecutive days before evaluation on D7 (P17).

Endothelial and Lymphocyte Cocultures. Blood was drawn from D7 rats with retinopathy and age-matched (P17) controls before sacrifice. Cytotoxic T lymphocytes (CTLs) were purified by positive selection via MACS using MicroBeads conjugated with a monoclonal anti-rat CD8a antibody (clone G-28; Miltenyi Biotec) according to the manufacturer's instructions. Human microvascular endothelial cells (Cascade Biologics, Inc.) at passage 6-8 were seeded at a concentration of $2 \times 10^{5}$ cells per well and stimulated with $30 \mathrm{ng} / \mathrm{ml}$ recombinant human tissue necrosis factor- $\alpha$ (TNF- $\alpha$; R\&D Systems). Isolated CTLs were incubated for $15 \mathrm{~min}$ at $37^{\circ} \mathrm{C}$ with $50 \mu \mathrm{M}$ carboxyfluorescein diacetate succinimidyl ester (CFDASE; Molecular Probes). The fluorescent cells were washed and incubated $\left(8 \times 10^{5}\right.$ cells $/ \mathrm{ml}, 100 \mu \mathrm{l}$ per well $)$ with endothelial monolayers for $4 \mathrm{~h}$, after which nonadherent lymphocytes were removed and the endothelial monolayer was washed. To examine whether the apoptosis is FasL-mediated, an anti-mouse FasL antibody (clone MFL4; BD Biosciences) or an isotype control antibody (BD Biosciences) was applied at $10 \mu \mathrm{g}$ / $\mathrm{ml}$ for $10 \mathrm{~min}$ at $37^{\circ} \mathrm{C}$ to the $\mathrm{T}$ cell suspension before the coculture. Cell death was assayed using the TUNEL procedure according to the manufacturer's instructions (Intergen). Apoptotic cells were detected using a CD-330 charge-coupled device camera (Dage-MIT) attached to an epifluorescence microscope (model MZ FLIII; Leica). A minimum of eight fields each in three separate experiments was analyzed per condition.

Morphometric and Statistical Analysis. All results were expressed as mean $\pm \mathrm{SD}$. The number of leukocytes in each flat mount was counted independently by two investigators under an epifluorescence microscope (DM RXA; Leica). The morphology of the pathological neovascularization was readily discerned from the intraretinal extension of physiological vessels. The neovascular retinal areas were photographed with an epifluorescence microscope (MZ FLIII; Leica) and measured using NIH Image. The values were processed for statistical analyses (Mann-Whitney $U$ test). Differences were considered statistically significant when the $\mathrm{P}$ values were $<0.05$.

\section{Results}

VEGF Expression in Physiological and Pathological Retinal Neovascularization. Absolute and relative VEGF isoform expression levels were studied during both physiological vascular development (Fig. 1, A and C) and pathological neovascularization (Fig. 1, B and D). ELISA results showed that retinal VEGF protein levels were approximately twofold higher in the pathologically neovascularizing retina (Fig. $1 \mathrm{~B}$ ) than in the developing retina undergoing physiological neovascularization (Fig. 1 A). RT-PCR showed a substantial relative difference in VEGF isoform expression pattern between pathological (Fig. 1 D) and physiological (Fig. $1 \mathrm{C}$ ) neovascularization. The $\mathrm{VEGF}_{164} / \mathrm{VEGF}_{120}$ expression ratio was calculated to be $2.2 \pm 1.1$ in the physio-
A normal retinal development

B proliferative retinopathy
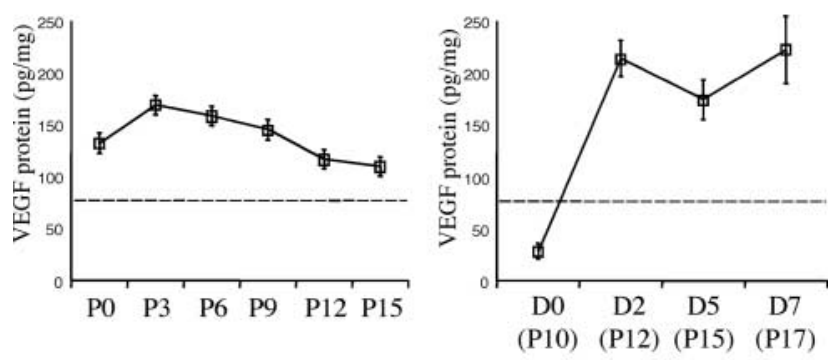

C normal retinal development

D proliferative retinopathy

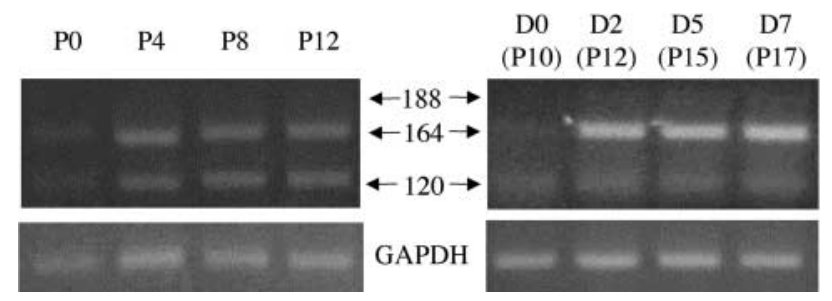

Figure 1. Differential expression of VEGF isoforms during physiologi$\mathrm{cal}$ and pathological retinal neovascularization. (A) Retinal VEGF protein levels (all isoforms) during postnatal development. (B) Retinal VEGF protein levels after the induction (D0) of pathological neovascularization. Dotted lines in A and B indicate the constitutive VEGF levels in normal adult rats. (C) Retinal VEGF mRNA expression during postnatal development. (D) Retinal VEGF mRNA expression after the induction of pathological neovascularization. The relative expression levels of $\mathrm{VEGF}_{164}$ increased dramatically during pathological neovascularization.

logically developing retinas, and $25.3 \pm 8.7$ in the pathologically neovascularized retinas.

Existence of Adherent Leukocytes at the Leading Edge of Pathological, but Not Physiological, Retinal Neovascularization. Leukocyte adhesion was examined at the leading edge of both physiological and pathological neovascularization. Adherent leukocytes were largely absent at the leading edge of physiological postnatal retinal vascular development (Fig. 2 A). In the retinopathy model of pathological neovascularization (Fig. 2, B-F), a vast area of avascular retina was formed (Fig. 2 B) during exposure to the hyperoxic environment (D0). Adherent leukocytes were found in the revascularizing retina (Fig. $2 \mathrm{C}$ ) on D2. These leukocytes were associated with the incipient pathological neovascular fronds (Fig. 2 D) on D3. By D7, leukocytes were observed in the areas of revascularization (Fig. $2 \mathrm{E}$ ), but were most prominent in the pathological neovascular fronds extending into the vitreous (Fig. $2 \mathrm{~F}$ ).

Effect of VEGF $164^{-s p e c i f i c ~ B l o c k a d e ~ o n ~ P h y s i o l o g i c a l ~ a n d ~}$ Pathological Retinal Neovascularization. A VEGF VE4(165) $_{160-}$ form-specific neutralizing aptamer (EYE001) was used to determine the role of $\mathrm{VEGF}_{164(165)}$ in pathological neovascularization. During pathological neovascularization (Fig. 3, A-F), VEGF ${ }_{164}$ blockade (Fig. 3 B) led to a significant inhibition of leukocyte adhesion (Fig. 3 D) and pathological neovascularization (Fig. 3 E). In contrast, little or no suppression of revascularization (Fig. $3 \mathrm{~F}$ ) and physiological neovascularization (Fig. 3, G, H, and J) was observed. 

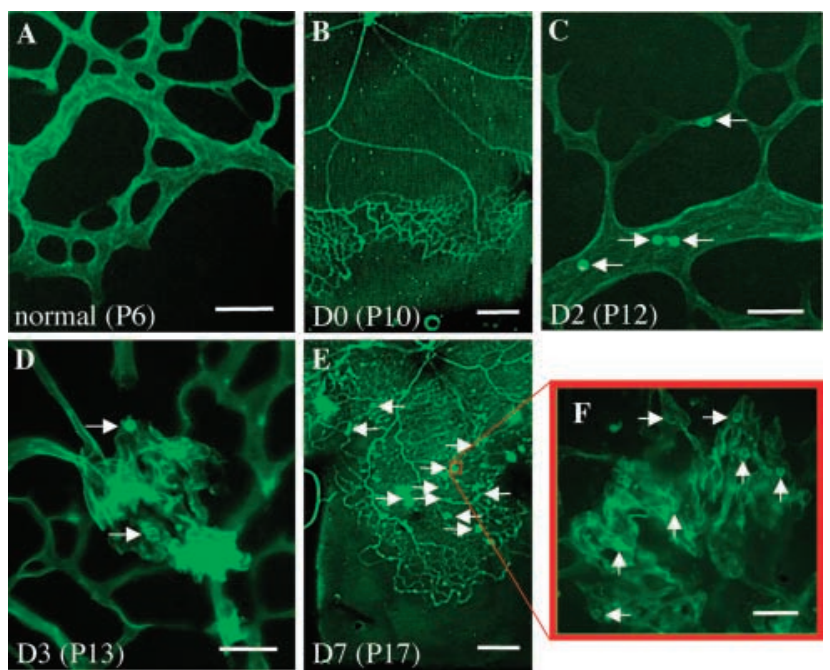

Figure 2. Leukocytes are present at the leading edge of pathological, but not physiological, retinal neovascularization. (A) No leukocyte adhesion at the leading edge of physiological retinal development on P6. (BF) Leukocyte adhesion during the onset and progression of pathological neovascularization. (B) The formation of extensive retinal ischemia on D0, immediately after exposure to relative hypoxia (room air). (C) Abundant inflammatory cells (arrows) randomly adhered to the intraretinal vessels just before the onset of pathological neovascularization. (D) The leukocyte adhesion (arrows) associated with the incipient vascular budding on D3. (E) Compared with D0 (B), revascularization proceeded in concert with pathological neovascularization (arrows) on D7. (F) Leukocyte adhesion (arrows) spatially and temporally associated with vascular budding. Bars: (A, C, D, and F) $50 \mu \mathrm{m}$; (B and E) $0.5 \mathrm{~mm}$.

When a VEGFR-1/Fc chimeric protein was used to compare the neutralizing effects of the single $\mathrm{VEGF}_{164}$ isoform versus all VEGF isoforms, VEGF pan-isoform blockade (Fig. $3 \mathrm{C}$ ) resulted in a significant suppression of leukocyte adhesion (Fig. $3 \mathrm{D}$ ) and pathological neovascularization (Fig. 3 E). The result was similar to that seen with $\mathrm{VEGF}_{164}$-specific blockade. However, in contrast to the $\mathrm{VEGF}_{164}$ inhibition result, a significant suppression of revascularization (Fig. $3 \mathrm{~F}$ ) and physiological neovascularization (Fig. 3, I and J) was observed with pan-isoform blockade. No difference was detected in physiological and pathological neovascularization among the following control groups: nontreated, PEG-treated, and Fc-treated rats (unpublished data). To further confirm the $\mathrm{VEGF}_{164}$ neutralizing effect on retinal vascular development, $\mathrm{VEGF}_{164^{-}}$ deficient mice were generated and evaluated. No difference in physiological neovascularization was detected between wild-type $\left(\mathrm{VEGF}^{+/+}\right)$mice and $\mathrm{VEGF}_{164}$-deficient $\left(V_{E G F}{ }^{120 / 188}\right)$ mice (Fig. 3, K-M).

Monocytes Are Positive Regulators of Pathological Retinal Neovascularization. To investigate the role of monocytes in pathological neovascularization, clodronate-liposomes were used to inactivate monocyte lineage cells. Compared with PBS-liposomes (Fig. 4 A), monocyte-selective depletion (Fig. 4 B) led to a significant suppression of pathological neovascularization (Fig. 4 C), but had negligible effect on revascularization (Fig. 4 D). Immunohistochemistry confirmed that a subset of the adherent leukocytes in the

vasculature (Fig. 4, E-J) were positive for CD13, aminopeptidase $\mathrm{N}$ coexpressed with MHC-II for monocyte-surface antigen processing. RT-PCR showed that circulating monocytes isolated from rats with proliferative retinopathy expressed $\mathrm{VEGF}_{120}$ and $\mathrm{VEGF}_{164}$, which was markedly induced after hypoxic stimulation (Fig. $4 \mathrm{~K}$ ).

T Lymphocytes Are Negative Regulators of Pathological Retinal Neovascularization. To investigate the role of $\mathrm{T}$ lymphocytes in pathological neovascularization, a neutralizing antibody against CD2 was used. CD2 is an important adhesion molecule for $\mathrm{T}$ lymphocyte-mediated immune responses, and is also called lymphocyte function-associated antigen-2 (LFA-2). Notably, compared with nonimmune control IgG (Fig. 5 A), CD2 blockade (Fig. 5 B) led to significant increase in the pathological neovascularization (Fig. $5 \mathrm{C})$. Immunohistochemistry confirmed that adherent leu-
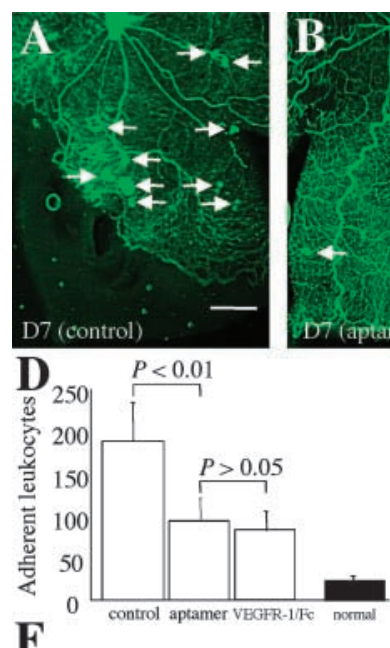

F
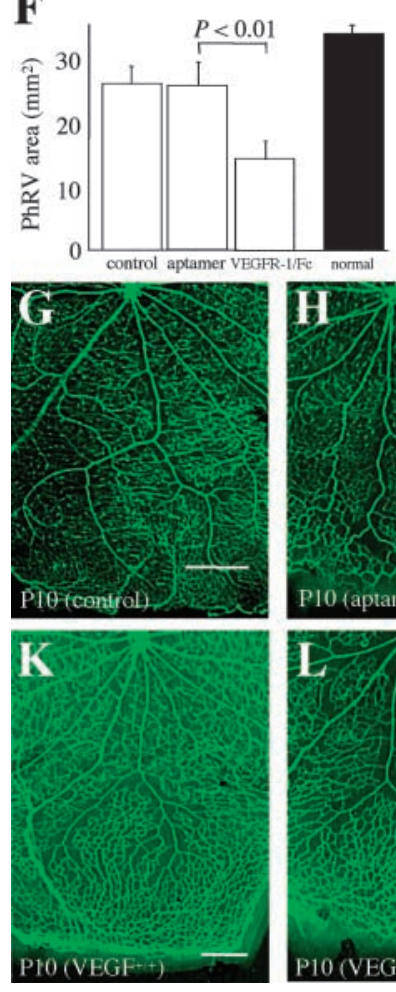
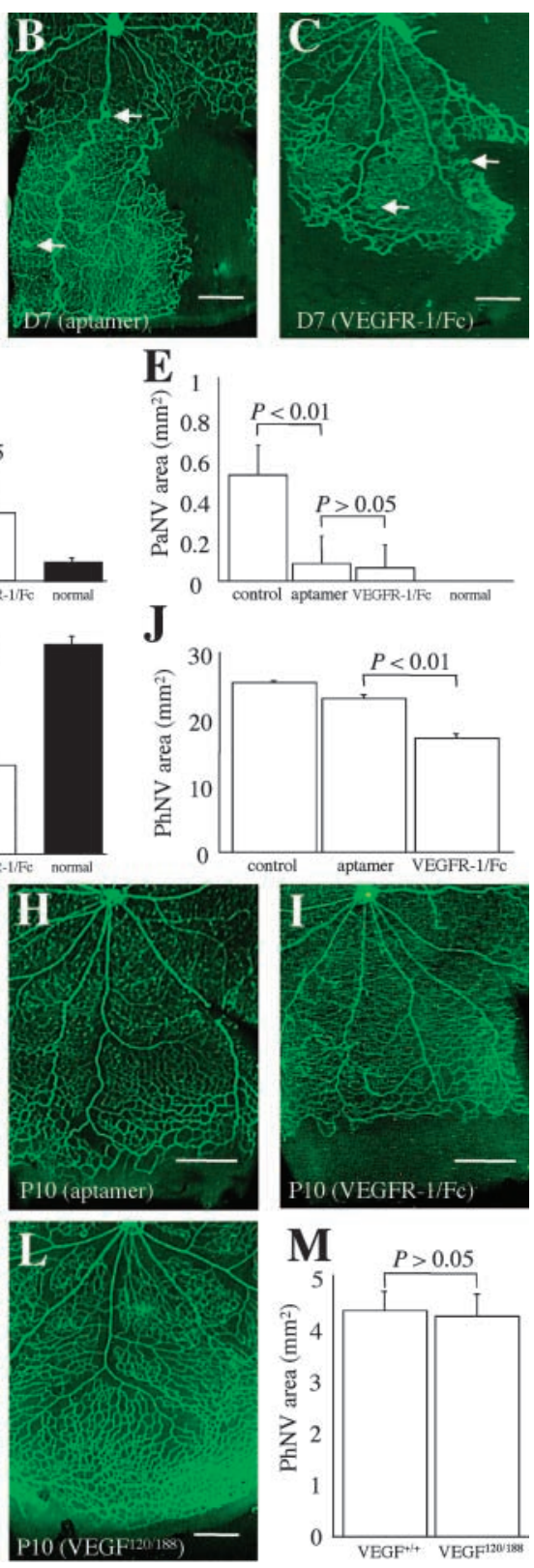
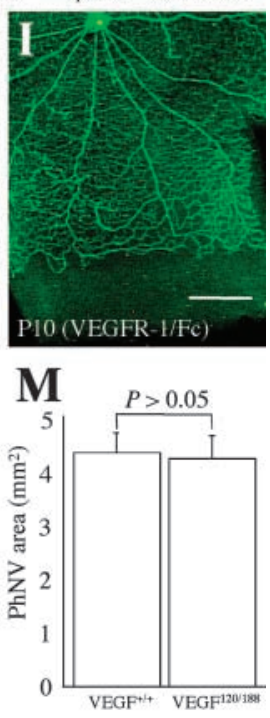

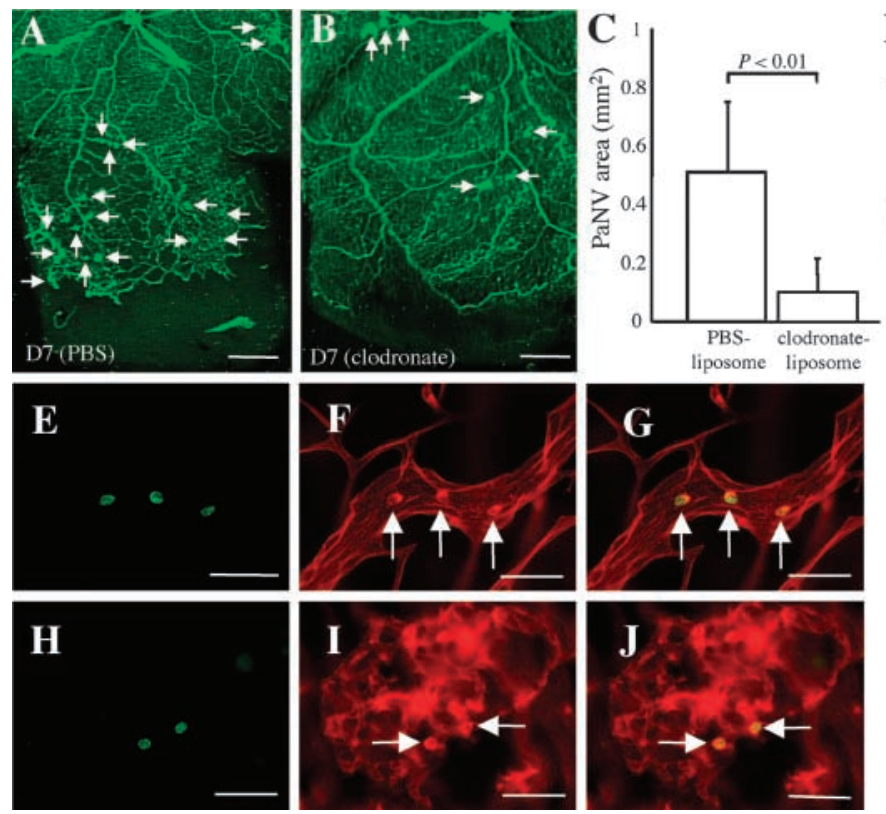

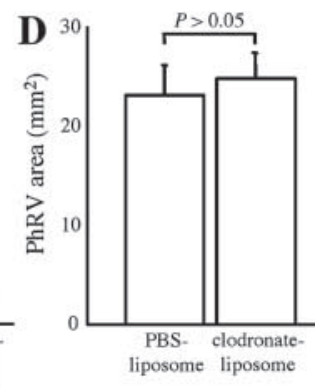

$\mathbf{K}$

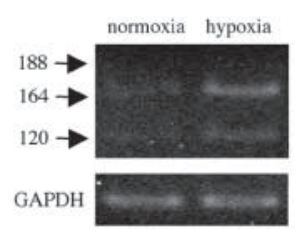

Figure 4. Role of monocytes in pathological retinal neovascularization. (A) Pathological neovascularization (arrows, D7) treated with PBS control-liposomes $(n=8)$ was not inhibited. (B) Pathological neovascularization (arrows, D7) treated with clodronate-liposomes $(n=8)$. Notably, the pathological neovascular budding (C, PaNV) was suppressed $(\mathrm{P}<0.01)$, whereas revascularization $(\mathrm{D}, \mathrm{phRV})$ was not $(\mathrm{P}>$ 0.05). (E-J) Monocyte adhesion was observed just before and during pathological neovascularization $(\mathrm{H}-\mathrm{J})$. Green fluorescence from the anti-CD13 antibody (E and $\mathrm{H}$ ) and red fluorescence from the rhodamine-coupled Con A (F and I) identifies the Con A-stained cells as being CD13positive leukocytes (arrows) when the images were superimposed ( $\mathrm{G}$ and $\mathrm{J})$. (K) Monocyte VEGF mRNA expression in normoxia (21\% oxygen) and hypoxia (1\% oxygen). VEGF levels were markedly increased in response to hypoxic stimulation. Bars: (E-J) $50 \mu \mathrm{m}$; (A and B) $0.5 \mathrm{~mm}$. kocytes at the vascular fronds were positive for CD8 (Fig. 5, D-F) and CD25 (Fig. 5, G-I; IL-2 receptor), indicative of cytotoxic and activated $\mathrm{T}$ lymphocytes, respectively. To determine whether CD8-positive cytotoxic T lymphocytes (CTLs) isolated from rats with retinopathy cause a FasLmediated endothelial cell apoptosis, cell death was evaluated using a leukocyte-endothelial coculture system. Compared with CTLs isolated from age-matched normal control rats (Fig. $5 \mathrm{~J}$ ), CTLs from rats with retinopathy (Fig. $5 \mathrm{~K}$ ) increased endothelial cell apoptosis (Fig. $5 \mathrm{~N}$ ).

Figure 3. Effect of $\mathrm{VEGF}_{164}$-specific blockade on physiological and pathological retinal neovascularization. (A-F) $\mathrm{VEGF}_{164}$-specific blockade versus VEGF pan-isoform blockade in pathological neovascularization. (A) Pathological neovascularization (arrows, D7) treated with PEG control $(n=12)$ was not inhibited. (B) Pathological neovascularization (arrows, D7) treated with the anti-VEGF ${ }_{165}$ aptamer $(n=13)$. (C) Pathological neovascularization (arrows, D7) treated with the VEGFR-1/Fc chimera $(n=10)$. In addition to the inhibition of leukocyte adhesion to the retinal vasculature $(\mathrm{D}, \mathrm{P}<0.01)$, pathological neovascular budding into the vitreous $(\mathrm{E}, \mathrm{PaNV})$ was significantly suppressed $(\mathrm{P}<0.01)$ via the anti$\mathrm{VEGF}_{164}$ aptamer or the VEGFR-1/Fc. In contrast, the effect of $\mathrm{VEGF}_{164}$ inhibition on physiological revascularization (F, PhRV) was negligible $(\mathrm{P}>0.05)$, but pan-isoform inhibition led to significant suppression of revascularization $(\mathrm{F}, \mathrm{P}<0.01)$. Shaded bars indicate comparable values of age-matched (P17) normal rat neonates $(n=8)$. (G-J) VEGF V64 $_{\text {-specific }}$ blockade versus VEGF pan-isoform blockade in retinal vascular development. (G) Developing retinal vasculature (P10) treated with PEG control $(n=7)$. (H) Developing retinal vasculature (P10) treated with anti$\operatorname{VEGF}_{165}$ aptamer $(n=8)$. (I) Developing retinal vasculature (P10) treated with the VEGFR-1/Fc chimera $(n=10)$. (J) Note the mild suppression of physiological neovascularization during retinal development via VEGF $_{164}$ inhibition, but the substantial suppression via pan-isoform inhibition $(\mathrm{P}<0.01)$. $(\mathrm{K}-\mathrm{M}) \mathrm{VEGF}_{164}$-specific deficiency versus wild type in retinal vascular development. $(\mathrm{K})$ Developing retinal vasculature (P10) in wild-type control $\left(\mathrm{VEGF}^{+/+}\right)$mice $(n=13)$. (L) Developing retinal vasculature (P10) in $\operatorname{VEGF}_{164}$-deficient $\left(\operatorname{VEGF}^{120 / 188}\right)$ mice $(n=13)$. (M) VEGF $_{164}$ deficiency had no significant effect on physiological neovascularization (P > 0.05). Bars: (K and L) $0.2 \mathrm{~mm}$; (A-C and G-I) $0.5 \mathrm{~mm}$.
Administration of a FasL neutralizing antibody (Fig. 5 L), but not a control nonimmune antibody (Fig. $5 \mathrm{M}$ ), led to significant suppression of the apoptosis caused by the CTLs isolated from the rats with retinopathy (Fig. $5 \mathrm{~N}$ ).

\section{Discussion}

The current data demonstrate that pathological, but not physiological, retinal neovascularization is characterized by the overexpression, both in relative and absolute terms, of the $\mathrm{VEGF}_{164}$ isoform. The hypoxia-induced $\mathrm{VEGF}_{164}$ isoform expression leads to the recruitment of leukocytes to sites of pathological neovascularization. These data demonstrate that ischemia-induced neovascularization, classically thought to be noninflammatory in nature, is characterized by the influx of inflammatory cells.

Monocyte/macrophage lineage cells express VEGF receptor (R)-1 through which VEGF exerts its chemotactic actions (6). VEGF also up-regulates the expression of ICAM-1 on vascular endothelial cells in vitro (7). VEGFinduced blood-retinal barrier breakdown was demonstrated to be leukocyte-dependent, in part, when the inhibition of ICAM-1 prevented blood-retinal barrier breakdown in $\mathrm{VEGF}_{165}$-injected rat eyes (8). In separate studies, VEGF-induced endothelial ICAM-1 expression (9) and vascular leakage (10) were shown to be mediated by VEGFR-2. Thus, VEGF functions as a proinflammatory cytokine targeting both leukocytes and endothelial cells. Taking into account the differential affinity of the VEGF isoforms for VEGF receptor binding $(11,12), \mathrm{VEGF}_{165}$ seemed likely to be more responsible for the induction of inflammation than VEGF $_{121}$.

The present work is the first to show that adherent leukocytes are associated with the leading edge of pathological, but not physiological, neovascularization (Fig. 2). The re- 

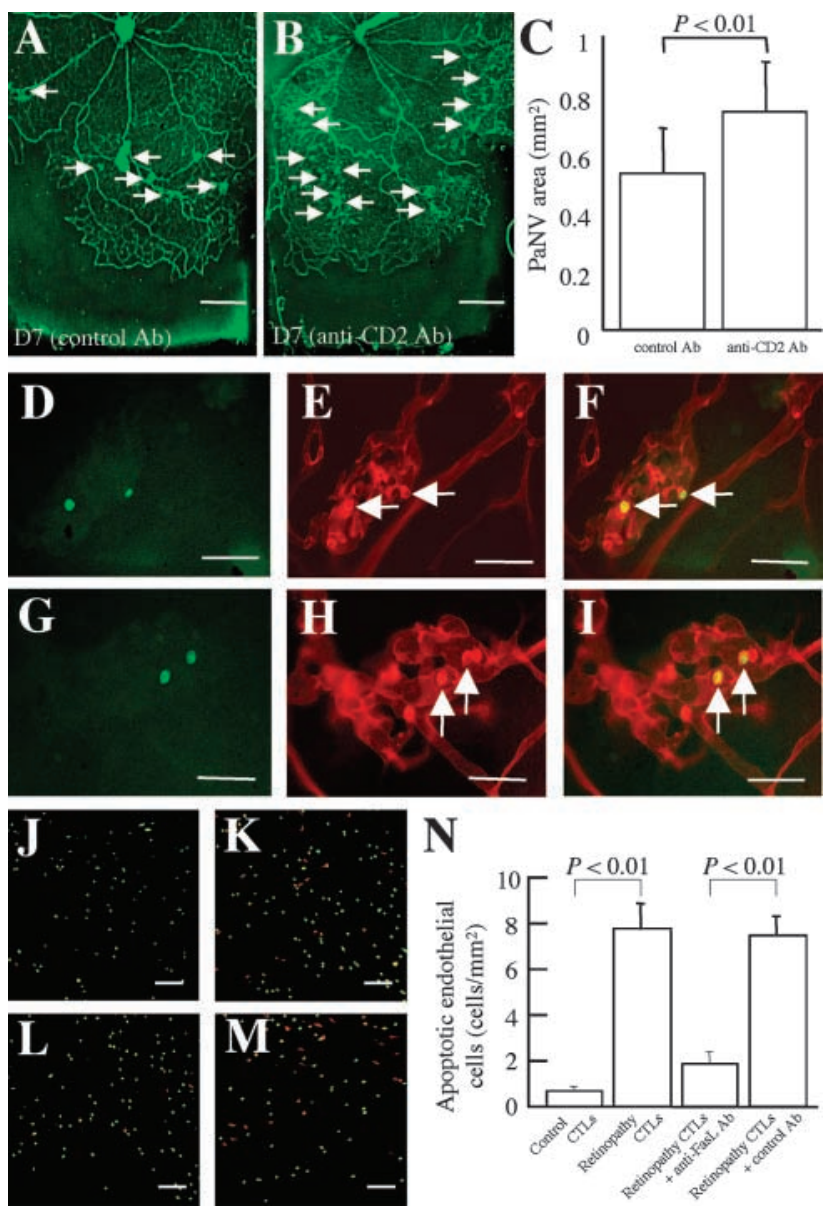

Figure 5. Role of $\mathrm{T}$ lymphocytes in pathological retinal neovascularization. (A) Pathological neovascularization (arrows, D7) treated with nonimmune isotype control $(n=9)$ showing a similar degree of pathological vascular budding (arrows) compared with Fig. 2 E. (B) Pathological neovascularization (arrows, D7) treated with anti-CD2 antibody $(n=$ 11). Notably, the pathological neovascular budding (C, PaNV) was worsened $(\mathrm{P}<0.01)$. (D-I) $\mathrm{T}$ cell subtypes in pathological neovascular buds. Green fluorescence from the antibody against CD8 or CD25 (D and G) and red fluorescence from the rhodamine-coupled Con $A$ (E and $H$ ) identifies the Con A-stained cells as being CD8- and CD25-positive leukocytes (arrows) when the images were superimposed ( $F$ and I). (J-N) Rhodamine-labeled apoptotic cells in the leukocyte-endothelial cocultures were detected via TUNEL staining (red). CTLs were labeled with CFDASE (green). Compared with control CTLs (J), retinopathy CTLs (K) significantly increased the number of TUNEL-positive endothelial cells $(\mathrm{N}, \mathrm{P}<0.01)$. The process was significantly inhibited via FasL blockade (L-N, $n=18-24$ in each condition, $\mathrm{P}<0.01)$. Bars: (D-I) 50 $\mu \mathrm{m}$; (J-M) $200 \mu \mathrm{m}$; and (A and B) $0.5 \mathrm{~mm}$.

cruitment of leukocytes was largely suppressed via $\mathrm{VEGF}_{164}$ isoform-specific blockade (Fig. 3), directly implicating $\mathrm{VEGF}_{164}$ in the process. Additionally, $\mathrm{VEGF}_{164}$, a more proinflammatory isoform than $\mathrm{VEGF}_{120}$, was preferentially induced in pathological neovascularization (Fig. 1). Together, these observations indicate that leukocyte adhesion in pathological, but not the physiological, neovascularization is properly regarded as a VEGF 164 -induced pathology.

The current data also show that the selective depletion of monocyte lineage cells in proliferative retinopathy sup- presses pathological neovascularization, but not revascularization (Fig. 4). The results mirror those seen after the $\mathrm{VEGF}_{164}$-specific blockade (Fig. 3). Thus, inflammatory monocytes appear to facilitate vitreous invasion by the aberrant vasculature. The $\mathrm{VEGF}_{164}$-mediated recruitment of monocytes just before the appearance of pathological neovascularization suggests that the monocytes may serve to mark the sites of future neovascularization (Fig. 2). Indeed, circulating monocytes have a pro-angiogenic potential expressing $\mathrm{VEGF}_{164}$, the production of which is markedly enhanced by hypoxia (Fig. 4). The positive feedback loop of $\mathrm{VEGF}_{164}$ and monocytes is a potential mechanism leading to the amplification of pathological neovascularization. Recent in situ hybridization data showing increased VEGF expression in the ischemic retinal cells (3) and macrophages (13) in the proliferative retinopathy model further support our conclusions.

$\mathrm{VEGF}_{164(165)}$, an isoform more potently mitogenic (11) as well as more proinflammatory than $\mathrm{VEGF}_{120(121)}$, appears to be sufficient for the physiological neovascularization of retinal development (5). However, VEGF isoforms other than $\mathrm{VEGF}_{164}$, in combination, may be sufficient to promote normal physiological neovascularization. Indeed, the complete inhibition of intracellular VEGF signaling was shown previously to result in substantial suppression of normal vascular development (3) and was reproduced here (Fig. 3), whereas $\mathrm{VEGF}_{164}$-selective blockade did not affect normal vascular development (Fig. 3). Consistent with these results, retinal vascular development was normal in genetically $\mathrm{VEGF}_{164}$-deficient mice expressing $\mathrm{VEGF}_{120}$ and $\mathrm{VEGF}_{188}$ (Fig. 3; VEGF ${ }^{120 / 188}$ mice) in agreement with the aptamerbased VEGF 164 neutralization data (Fig. 3). Consequently, the expression pattern of the various VEGF isoforms, strictly regulated during normal development but disrupted in disease, is likely to contribute to an angiogenic switch, converting the orderly intraretinal extension of vessels into an inflammation-associated invasion of the vitreous.

The present paper shows that IL-2 receptor (CD25)positive cells and CD8-positive cells adhere to the pathological neovascular fronds (Fig. 5), indicative of the presence of activated CTLs. In a murine tumor model, the systemic administration of IL-12 and IL-2, both known to activate cellular immunity, led to the recruitment of FasLpositive CTLs and the inhibition of tumor neovascularization and growth (14). Conversely, an angiogenic role of T lymphocytes, a source of VEGF, has been suggested in human prostate cancer (15). The present paper demonstrates that the inhibition of lymphocyte function results in the aggravation of pathological neovascularization (Fig. 5). In our model of pathological retinal neovascularization, CTLmediated cellular immunity is thought to play a critical role in the defense against the ectopic proliferation of endothelial cells. This is also supported by the findings that CD8positive $\mathrm{T}$ lymphocytes from rats undergoing pathological neovascularization resulted in the FasL-mediated apoptosis of endothelial cells in vitro (Fig. 5). The role of immunotherapy for retinal neovascular diseases is currently under investigation. 
This work was funded by Eyetech Pharmaceuticals. Drs. Adamis and Shima are employees and shareholders of Eyetech Pharmaceuticals. The anti-VEGF ${ }_{165}$ aptamer used in these experiments is a product of Eyetech Pharmaceuticals and is currently in clinical trial.

Submitted: 21 November 2002

Revised: 13 May 2003

Accepted: 23 May 2003

\section{References}

1. Stone, J., A. Itin, T. Alon, J. Pe'er, H. Gnessin, T. ChanLing, and E. Keshet. 1995. Development of retinal vasculature is mediated by hypoxia-induced vascular endothelial growth factor (VEGF) expression by neuroglia. J. Neurosci. 15:4738-4747.

2. Stone, J., T. Chan-Ling, J. Pe'er, A. Itin, H. Gnessin, and E. Keshet. 1996. Roles of vascular endothelial growth factor and astrocyte degeneration in the genesis of retinopathy of prematurity. Invest. Ophthalmol. Vis. Sci. 37:290-299.

3. Ozaki, H., M.S. Seo, K. Ozaki, H. Yamada, E. Yamada, N. Okamoto, F. Hofmann, J.M. Wood, and P.A. Campochiaro. 2000. Blockade of vascular endothelial cell growth factor receptor signaling is sufficient to completely prevent retinal neovascularization. Am. J. Pathol. 156:697-707.

4. Joussen, A.M., T. Murata, A. Tsujikawa, B. Kirchhof, S.E. Bursell, and A.P. Adamis. 2001. Leukocyte-mediated endothelial cell injury and death in the diabetic retina. Am. J. Pathol. 158:147-152.

5. Stalmans, I., Y.S. Ng, R. Rohan, M. Fruttiger, A. Bouche, A. Yuce, H. Fujisawa, B. Hermans, M. Shani, S. Jansen, et al. 2002. Arteriolar and venular patterning in retinas of mice selectively expressing VEGF isoforms. J. Clin. Invest. 109:327336.

6. Barleon, B., S. Sozzani, D. Zhou, H.A. Weich, A. Mantovani, and D. Marme. 1996. Migration of human monocytes in response to vascular endothelial growth factor (VEGF) is mediated via the VEGF receptor flt-1. Blood. 87:3336-3343.

7. Melder, R.J., G.C. Koenig, B.P. Witwer, N. Safabakhsh, L.L. Munn, and R.K. Jain. 1996. During angiogenesis, vascular endothelial growth factor and basic fibroblast growth factor regulate natural killer cell adhesion to tumor endothelium. Nat. Med. 2:992-997.
8. Miyamoto, K., S. Khosrof, S.E. Bursell, Y. Moromizato, L.P. Aiello, Y. Ogura, and A.P. Adamis. 2000. Vascular endothelial growth factor (VEGF)-induced retinal vascular permeability is mediated by intercellular adhesion molecule-1 (ICAM-1). Am. J. Pathol. 156:1733-1739.

9. Kim, I., S.O. Moon, S.H. Kim, H.J. Kim, Y.S. Koh, and G.Y. Koh. 2001. Vascular endothelial growth factor expression of intercellular adhesion molecule 1 (ICAM-1), vascular cell adhesion molecule 1 (VCAM-1), and E-selectin through nuclear factor-kappa B activation in endothelial cells. J. Biol. Chem. 276:7614-7620.

10. Gille, H., J. Kowalski, B. Li, J. LeCouter, B. Moffat, T.F. Zioncheck, N. Pelletier, and N. Ferrara. 2001. Analysis of biological effects and signaling properties of Flt-1 (VEGFR-1) and KDR (VEGFR-2). A reassessment using novel receptorspecific vascular endothelial growth factor mutants. J. Biol. Chem. 276:3222-3230.

11. Keyt, B.A., L.T. Berleau, H.V. Nguyen, H. Chen, H. Heinsohn, R. Vandlen, and N. Ferrara. 1996. The carboxyl-terminal domain (111-165) of vascular endothelial growth factor is critical for its mitogenic potency. J. Biol. Chem. 271:77887795.

12. Whitaker, G.B., B.J. Limberg, and J.S. Rosenbaum. 2001. Vascular endothelial growth factor receptor-2 and neuropilin-1 form a receptor complex that is responsible for the differential signaling potency of $\operatorname{VEGF}(165)$ and $\operatorname{VEGF}(121) . J$. Biol. Chem. 276:25520-25531.

13. Naug, H.L., J. Browning, G.A. Gole, and G. Gobe. 2000. Vitreal macrophages express vascular endothelial growth factor in oxygen-induced retinopathy. Clin. Exp. Ophthalmol. $28: 48-52$

14. Wigginton, J.M., E. Gruys, L. Geiselhart, J. Subleski, K.L. Komschlies, J.W. Park, T.A. Wiltrout, K. Nagashima, T.C. Back, and R.H. Wiltrout. 2001. IFN-gamma and Fas/FasL are required for the antitumor and antiangiogenic effects of IL-12/pulse IL-2 therapy. J. Clin. Invest. 108:51-62.

15. Freeman, M.R., F.X. Schneck, M.L. Gagnon, C. Corless, S. Soker, K. Niknejad, G.E. Peoples, and M. Klagsbrun. 1995. Peripheral blood $\mathrm{T}$ lymphocytes and lymphocytes infiltrating human cancers express vascular endothelial growth factor: a potential role for $\mathrm{T}$ cells in angiogenesis. Cancer Res. 55: 4140-4145. 